\title{
Is Intra-Articular Administration of Fibrinogen Effective in Postoperative Total Knee Arthroplasty Blood Loss? A Randomized Clinical Trial
}

\author{
Farahzad Janatmakan $\mathbb{D}^{1,{ }^{1},}$, Fatemeh Javaherforooshzadeh ${ }^{2, * *}$, Mohsen Khorrami $^{3}$, Sara Jarirahmadi ${ }^{2}$ \\ and Hojjatollah Khademali ${ }^{2}$ \\ ${ }^{1}$ Department of Anesthesia, Pain Research Center, Ahvaz Jundishapur University of Medical Sciences, Ahvaz, Iran \\ ${ }^{2}$ Department of Anesthesia, Ahvaz Anesthesiology and Pain Research Center, Ahvaz Jundishapur University of Medical Sciences, Ahvaz, Iran \\ ${ }^{3}$ Department of Orthopedic Surgery \& Trauma Research Center, Ahvaz Jundishapur University of Medical Sciences, Ahvaz, Iran \\ "Corresponding author: Department of Anesthesia, Pain Research Center, Ahvaz Jundishapur University of Medical Sciences, Ahvaz, Iran. Email: fjanatmakan@yahoo.com \\ ${ }^{* *}$ Corresponding author: Department of Anesthesia, Ahvaz Anesthesiology and Pain Research Center, Ahvaz Jundishapur University of Medical Sciences, Ahvaz, Iran. Email: \\ f_javaherforoosh@yahoo.com
}

Received 2020 August 15; Revised 2020 November 18; Accepted 2020 November 18.

\begin{abstract}
Objectives: The purpose of this study was to evaluate the effect of intra-articular injection of fibrinogen on postoperative bleeding following total knee arthroplasty.

Methods: A double-blind randomized clinical trial was conducted on 40 patients aged 40 - 70 years under spinal anesthesia candidate for total knee arthroplasty in Golestan hospital, Ahwaz, Iran, in 2017-2018. Patients were divided into fibrinogen intra-articular injection $(n=20)$ and control $(n=20)$ groups. The amounts of blood loss and blood transfusion requirement were recorded. Hemoglobin ( $\mathrm{Hb}$ ), hematocrit (HCT), international normalized ratio (INR), platelet (PLT), prothrombin time (PT), and partial thromboplastin time (PTT) were recorded before and after the surgery.

Results: There was no significant difference in the average amount of intraoperative blood loss between the groups ( $\mathrm{P}>0.05)$. The average amount of blood loss 24 hours after the surgery was significantly lower in the fibrinogen group than in the control group (fibrinogen group $350.61 \pm 120.32 \mathrm{cc}$; control group $540.00 \pm 170.21 \mathrm{cc} ; \mathrm{P}=0.0002$ ). There were significant differences in transfusion between the groups (fibrinogen group $250 \pm 20 \mathrm{cc}$; control group $350 \pm 50 \mathrm{cc} ; \mathrm{P}<0.0001$ ). There was a significant difference between the two groups in 24 h postoperative $\mathrm{Hb}$ and $\mathrm{HCT}(\mathrm{P}<0.001)$.

Conclusions: Intra-articular fibrinogen administration may reduce acute bleeding and can be used as an effective intervention to prevent further bleeding and the need for transfusion in patients undergoing total knee arthroplasty.
\end{abstract}

Keywords: Fibrinogen, Intra-Articular Injection, Total Knee Arthroplasty, Blood Loss

\section{Background}

Total knee arthroplasty (TKA) is a common surgical procedure for the treatment of patients with severe arthritis. One of the complications of TKA is major bleeding, as $10-38 \%$ of patients would require blood transfusion (1). The volume of bleeding is sometimes $1,500 \mathrm{~mL}$ (800 $1800)$ on average $(2,3)$. Postoperative bleeding can cause hematoma, pain, limitation of knee movement, and delayed wound healing. Also, reducing bleeding is important to maintain hemodynamic stability for the patient and reduce complications such as hemolytic and non-hemolytic reactions, acute injury, viral and bacterial infections, hypothermia, and coagulopathy (4). The Zhang et al. study demonstrated that after the release of the tourniquet, fib- rinolysis is activated at the location, increasing the blood loss (5). Therefore, it is important to find a method to reduce bleeding during surgery (6).

Various methods can be effective in reducing perioperative hemorrhage, including intraoperative controlled hypotension, tourniquet use, intravenous and local use of anti-fibrinolytic drugs, fibrin sealants, and autologous platelet gels $(7,8)$. Fibrinogen is an anti-fibrinolytic agent that plays a key role in the formation, reinforcement, and strengthening of the clot (9). Fibrinogen is a plasma glycoprotein made by the liver, which is converted to fibrin and blood clots by thrombin enzymes following tissue and vascular injury. It is a substrate for thrombin, plasmin, and active factor XIII, with a half-life of three to five days (10). 
Hypofibrinogenemia occurs due to factors such as hemorrhage, hemodilution, consumption due to blood clotting, and Disseminated Intravascular Coagulation (DIC) (11). Thus, it is important to use fibrinogen to keep its blood concentration at the normal level and control clot formation in patients with hemorrhage (12). The normal level of fibrinogen is 200 to $450 \mathrm{mg} / \mathrm{dL}$ (13).

There are different methods for administering fibrinogen. A common way is an intravenous administration. In this route of administration, the drug is distributed throughout the body, but an inadequate amount of the drug may be provided for the injured tissue (14). The complications of intravenous fibrinogen injection include allergic reactions, fever, and thrombotic events such as myocardial infarction and pulmonary embolism (14-17). Another method of prescribing fibrinogen is homeostatic patches, lengthways with collagen, and thrombin. The complications of this method are immune responses and renal complications $(18,19)$. The topical administration of fibrinogen is a new approach for the administration of this drug. This approach is associated with lower drug requirements and complications and more effectiveness. This method has been described to stop diffuse hemorrhage in TKA in small studies and could be a hopeful approach to reduce blood loss and lower transfusion rates (20).

There are several opinions on the administration of intra-articular fibrinogen. In the study of Heyse et al., the intra-articular administration of fibrinogen in TKA did not lead to a significant reduction in blood loss and transfusion in primary TKA for osteoarthritis (21). Bae et al. showed that a thrombin-based hemostatic agent was effective in reducing drainage and blood transfusion in TKA (22). Due to the contradictory results regarding the intra-articular administration of fibrinogen, we decided to conduct a study to investigate the effect of intra-articular injection of fibrinogen on the amount of bleeding during TKA.

\section{Objectives}

Due to the contradictory results regarding the intraarticular administration of fibrinogen, we decided to conduct a study to investigate the effect of intra-articular injection of fibrinogen on the amount of bleeding during TKA.

\section{Methods}

This study was approved by the Ethics Committee of Ahvaz Jundishapur University of Medical Sciences (IR.AJUMS.REC.1397.419) and registered at IRCT (IRCT20190222042803N1). This double-blind randomized clinical trial was conducted on 40 patients aged $40-70$ years, with ASA Class I and II, under spinal anesthesia, candidate for TKA in Golestan hospital, Ahwaz, Iran, in 2017 2018. Patients were informed of the goals of the study and gave their written consent. The exclusion criteria were dissatisfaction with participation in the study, a history of arthroscopic knee surgery, uncontrolled hypertension, cardiovascular disease, deep venous thrombosis, coagulopathy, use of NSAID and anticoagulant drugs, body mass index $>30$, and operation time $>120 \mathrm{~min}$.

\subsection{Randomization}

The patients were randomized 1:1 to take either fibrinogen or placebo. To ensure that patients, surgeons, and investigators were blind to the treatment before the study, the patient enrollment was done based on computergenerated allocation concealment. The sequences were generated by a self-governing calculator using a random number generator with 1:1 allocation and random block sizes of two.

\subsection{Blindness}

To blind the study, the patients, the surgeon, and the investigator were not aware of the type of the drug, and the surgeries were performed by a single surgeon. The injectable drug was prepared by the researcher and named A and $\mathrm{B}$.

\subsection{Sample Size}

The sample size was calculated by the sample size estimation formula based on a previous study (23). Considering a probable mean difference of 225 cc or greater in total bleeding between the two groups, to find a statistical power of 0.80 at an Alfa error of 0.05, 20 patients were required in each group. By considering that $10 \%$ of the patients would be omitted, 30 patients were enrolled in each group. During the study period from December 2017 to November 2018, 65 patients undergoing elective TNA surgery were qualified to contribute to the trial. They were agreed to contribute and gave their written informed consent. Among them, 10 patients did not meet the inclusion criteria (seven patients had ASA $>2$, and three patients were aged $>70$ ). Three patients had a history of cardiovascular disease, five patients were giving anticoagulant drugs, five patients had a body mass index $>30$, and two patients had operation time $>120 \mathrm{~min}$. Finally, 40 patients were registered in the study and allocated to the two groups of fibrinogen and control, each with 20 patients (CONSORT diagram).

Patients were divided into two groups: fibrinogen (n $=20)$ and control $(n=20)$. Hemoglobin $(\mathrm{Hb})$, hematocrit (HCT), international normalized ratio(INR), platelets (PLT), 
prothrombin time (PT), and partial thromboplastin time (PTT) were recorded before the surgery as baseline data. Before spinal anesthesia, routine monitoring (pulse oximetry, non-invasive blood pressure, and ECG) was performed, and a ringer solution of $10 \mathrm{~mL} / \mathrm{kg}$ was infused. Spinal anesthesia was performed in the patients in a sitting position through the L4 - L5 or L3 - L4 spaces using 26G Quincke spinal needles. Hyperbaric bupivacaine $(12.5 \mathrm{mg})$ was injected after assuring proper subarachnoid space detection. In the first 10 minutes, the blood pressure was measured every three minutes, and then it was measured every $5 \mathrm{~min}$. If the systolic blood pressure decreased by $25 \%$ of baseline or below $90 \mathrm{mmHg}, 5 \mathrm{mg}$ of intravenous ephedrine was injected. The patient's sensory level was assessed by the pinprick method, and at the T6 level, the surgery was allowed. Tourniquets were used for all patients. The amount of intraoperative bleeding in both groups was calculated based on the difference between the total suction volume and the known irrigation volume.

About two minutes before releasing the tourniquet pressure, $500 \mathrm{mg}$ fibrinogen (Evicel; Ethicon, Johnson \& Johnson, Somerville, NJ, USA) diluted with normal saline at a total volume of $20 \mathrm{ml}$, for patients in the fibrinogen group, and $20 \mathrm{~mL}$ of normal saline, for patients in the control group, were injected locally to the knee joint by the surgeon (24). All patients underwent posterior stabilized TKA (Zimmer IN, USA, or Legion, Smith \& Nephew, Memphis, TN, USA). At the end of the surgery, one deep drain was fixed for each patient (3.175-mm ConstaVac; Stryker, Kalamazoo, MI, USA) and was placed in low-pressure suction $(25 \mathrm{mmHg}$ ) for $24 \mathrm{~h}$. The bleeding was calculated and compensated with a packed cell. The estimation of the total blood loss was done by a mathematical approach. We used Nadler's formula for calculating the blood volume (25), and then, the approximate blood loss was determined by using Mercuriali and Rosechner's formula (26). The start point for transfusion was a hemoglobin level $<8$ $\mathrm{g} / \mathrm{dL}$ unless medical causes required transfusion above this level. If the amount of bleeding was less than ABL, the patient was given $3 \mathrm{~mL}$ of isotonic serum per one milliliter of blood loss. After releasing the tourniquet, the site of operation was sutured and dressed, and patients were transferred to the recovery room. The primary outcomes were intraoperative bleeding, total 24-h drainage collected in the drain, and postoperative blood transfusion. The secondary outcomes were Hb, HCT, INR, PLT, PT, and PTT before the surgery and 24 hours after the surgery.

\subsection{Statistical Analysis}

Numeric data were described as mean \pm standard deviation (SD), percentages (\%), and numbers ( $\mathrm{n}$ ). The independent t-test, Mann-Whitney test, and chi-square test were used for quantitative and qualitative variables. The P values of $\leq 0.05$ were considered statistically significant. All analyses were performed using SPSS version 22.0 (SPSS Inc., Chicago, IL, USA).

\section{Results}

There were no significant differences between the groups in demographic characteristics (age, sex, and weight) and surgical data (tourniquet time and underlying disease) (Table 1).

Concerning the primary outcomes, the intraoperative bleeding was lower in the fibrinogen group, but there was no significant difference $(P=0.247)$ (Table 2$)$. The average amount of blood loss 24 hours after surgery was significantly lower in the fibrinogen group than in the control group (fibrinogen group: $350.61 \pm 120.32 \mathrm{cc}$; control group $540.00 \pm 170.21 \mathrm{cc} ; \mathrm{P}=0.0002$ ). There were significant differences in transfusion between the groups (fibrinogen group $250 \pm 20 \mathrm{cc}$; control group $350 \pm 50 \mathrm{cc}$; P $<0.0001$ ) (Table 2).

Concerning the secondary outcomes, there was a significant difference between the groups in $24 \mathrm{~h}$ postoperative $\mathrm{Hb}$ and HCT $(\mathrm{P}<0.001)$ (Figure 1). The mean platelet, PT, PTT, INR, and HCT levels before the surgery were not different between the two groups $(P>0.05)$. After 24 hours of the completion of the surgery, the average blood platelet count was reported to be higher in the fibrinogen group, but there was no significant difference $(P>0.05)$. Besides, PT increased in the control group, but there was no significant difference $(P>0.05)$. However, PTT and INR increased significantly after the surgery in the control group $(\mathrm{P}<0.05)$ (Table 3$)$.

\section{Discussion}

Bleeding after TKA can increase the need for blood transfusion, which enhances the costs and risks of the surgery, and is associated with some complications (27). The administration of topical fibrinogen can reduce drainage, decrease hemoglobin, and lower the need for blood transfusion in TKA. In the present study, although the mean volume of bleeding during surgery was lower in the fibrinogen group than in the control group, there was no significant difference $(P>0.05)$. However, the mean volume of postoperative bleeding was significantly lower in the fibrinogen group than in the control group $(\mathrm{P}=$ 0.0002). In a study, Heyse et al. concluded that the use of fibrinogen in TKA did not lead to a significant decrease in blood loss or transfusion in TKA for osteoarthritis (21). In their study, fibrinogen was used inside the medial 


\begin{tabular}{|c|c|c|c|}
\hline Variables & Fibrinogen Group $(\mathrm{N}=\mathbf{2 0})$ & Control Group $(\mathrm{N}=\mathbf{2 0})$ & P-Value \\
\hline $\operatorname{Age}(y)$ & $60.25 \pm 3.70$ & $59.4 \pm 3.97$ & 0.513 \\
\hline $\operatorname{Sex}(M / F)$ & $8 / 12$ & $6 / 14$ & 0.401 \\
\hline Weight (Kg) & $73.63 \pm 6.48$ & $76.67 \pm 6.31$ & 0.141 \\
\hline Tourniquet time (min) & $84.3(58-110)$ fisher & $91.1(60-115)$ & 0.391 \\
\hline Preoperative hematocrit (\%) & $36.81 \pm 4.26$ & $36.20 \pm 4.31$ & 0.658 \\
\hline Preoperative hemoglobin $(\mathrm{g} / \mathrm{dL})$ & $12.49 \pm 1.83$ & $12.11 \pm 1.47$ & 0.477 \\
\hline \multicolumn{4}{|c|}{$\begin{array}{l}\text { a Data are expressed as mean } \pm \text { standard deviation. } \\
\text { b Group comparisons were made using t-tests and Fischer's Exact Test (tourniquet time comparison between two groups). }\end{array}$} \\
\hline \multicolumn{4}{|c|}{ ble 2. Primary Outcomes in Both Groups of Patients ${ }^{a, b}$} \\
\hline Variables & Fibrinogen Group $(\mathrm{N}=\mathbf{2 0})$ & Control Group $(\mathrm{N}=\mathbf{2 0})$ & P-Value \\
\hline Intraoperative blood loss (cc) & $67.50 \pm 26.53$ & $76.50 \pm 24.77$ & 0.247 \\
\hline Blood loss in 24 hours (cc) & $350.61 \pm 120.32$ & $540.00 \pm 170.21$ & 0.0002 \\
\hline Transfusion (cc) & $250 \pm 20$ & $350 \pm 50$ & $<0.0001$ \\
\hline \multicolumn{4}{|c|}{$\begin{array}{l}{ }^{\mathrm{a}} \text { Data are expressed as mean } \pm \text { standard deviation. } \\
{ }^{\mathrm{b}} \text { Group comparisons were made using t-tests. }\end{array}$} \\
\hline
\end{tabular}

A

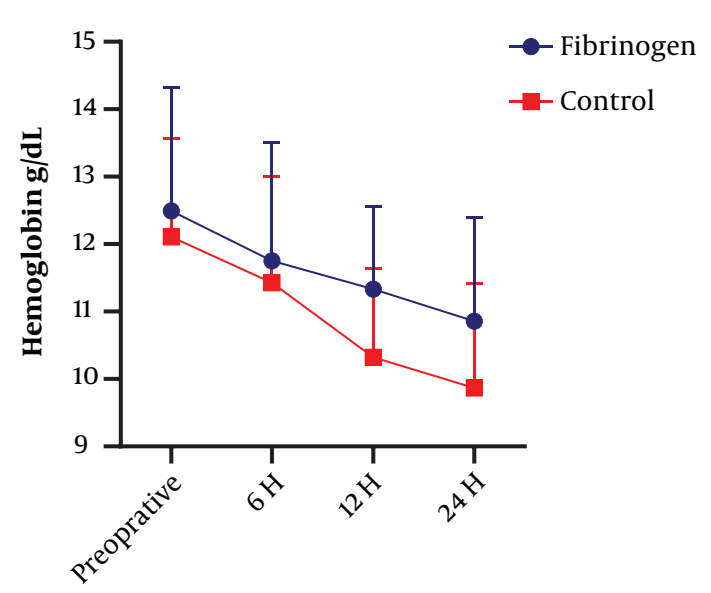

B

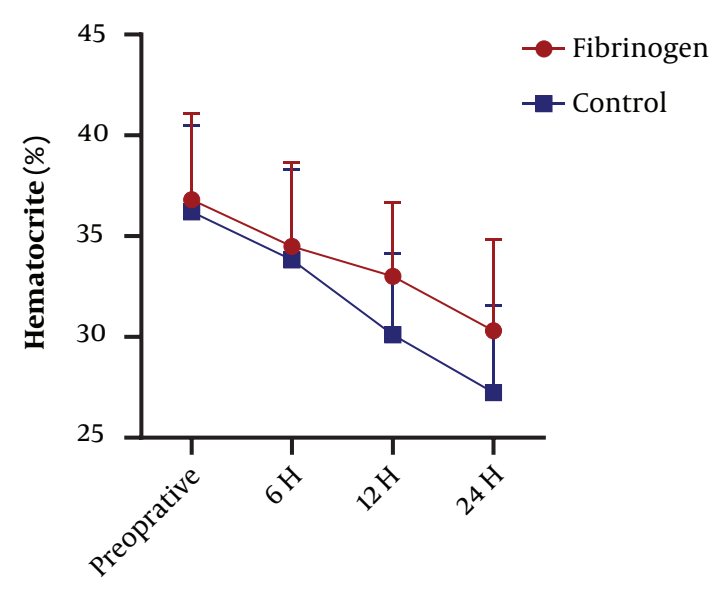

Figure 1. Average 24-hour Hgb (A) and Hct (B) preoperatively and perioperatively in fibrinogen and control groups.

knee joint space but did not significantly reduce bleeding and blood transfusion. This difference with our results might be attributed to the method of cauterization applied after the release of the tourniquet. There are two previous studies about the use of fibrinogen for blood loss control in TKA. Levy et al. carried out a prospective, controlled, randomized study on 58 patients undergoing TKA using a human fibrin tissue adhesive from a different manufacturer (Quixil; Omrix Biopharmaceuticals SA, NesZiona, Israel). They used the cruciate-retaining knees and low-molecular-weight heparin for thromboprophylaxis. Their study recognized significant benefits in the intervention group concerning postoperative drain output, hemoglobin drop, and transfusion rate (28). Their results were comparable to this study, although they used fibrin tissue adhesive. The study by Wang et al. described similar results with the same product in patients undergoing TKA in a cohort study (29). Quixil contains tranexamic acid, which has been confirmed to be effective in reducing blood loss. 


\begin{tabular}{|c|c|c|c|}
\hline Blood Factors & Fibrinogen Group $(\mathrm{N}=\mathbf{2 0})$ & Control Group $(\mathrm{N}=\mathbf{2 0})$ & P-Value \\
\hline \multicolumn{4}{|l|}{ Platelets } \\
\hline Baseline & $312.85 \pm 98.680$ & $306.40 \pm 67.786$ & 0.811 \\
\hline After Surgery & $291.05 \pm 94.009$ & $278.75 \pm 65.754$ & 0.634 \\
\hline \multicolumn{4}{|l|}{ PT } \\
\hline Baseline & $13.315 \pm 1.128$ & $12.885 \pm 0.829$ & 0.178 \\
\hline After Surgery & $13.675 \pm 1.378$ & $13.455 \pm 0.871$ & 0.550 \\
\hline \multicolumn{4}{|l|}{ PTT } \\
\hline Baseline & $30.150 \pm 2.66$ & $30.00 \pm 1.41$ & 0.825 \\
\hline After Surgery & $32.40 \pm 2.18$ & $33.05 \pm 2.84$ & 0.442 \\
\hline \multicolumn{4}{|l|}{ INR } \\
\hline Baseline & $0.99 \pm 0.09$ & $0.98 \pm 0.12$ & 0.740 \\
\hline After Surgery & $1.09 \pm 0.07$ & $1.13 \pm 0.11$ & 0.174 \\
\hline \multicolumn{4}{|l|}{ HCT } \\
\hline Baseline & $36.81 \pm 4.26$ & $36.20 \pm 4.31$ & 0.658 \\
\hline After Surgery & $34.49 \pm 4.17$ & $33.82 \pm 4.49$ & 0.628 \\
\hline
\end{tabular}

${ }^{\mathrm{a}}$ Data are expressed as mean \pm standard deviation.

${ }^{\mathrm{b}}$ The statistical test was the t-test.

In a study, Bae et al. evaluated the hemostatic effect of intra-articular injection of a thrombin-based hemostatic agent in TKA. They showed the efficacy of this agent in reducing drain output and blood transfusion rates (22). This study was in line with our study concerning drain output and HB decrease. In 2016, the results of a study by Tartaglia et al. on patients undergoing thyroid surgery indicated that the use of collagen-fibrinogen-thrombin patch reduced the amount of postoperative drainage, as well as bleeding-related complications (30). The Payani et al. study used topical fibrinogen on the precordium in open cardiac surgery. They indicated a reduction in postoperative blood loss after CABG, and patients had higher hematocrit in the postoperative period (31). A recent study obtained similar results, demonstrating that in TNA, the local injection of fibrinogen could reduce postoperative bleeding.

\subsection{Limitations}

The limitations of this study were the small number of patients so that with this number of cases, we could not get the real result. It is recommended that future studies be conducted with a larger sample size in multiple centers.

\subsection{Conclusion}

The results of this study showed that intra-articular injection of fibrinogen may play an important role in reducing acute postoperative hemorrhage and can be used as an effective intervention to prevent further bleeding and reduce the need for blood products in patients undergoing TNA.

\section{Acknowledgments}

This research was issued from a thesis by Dr. Hojjatollah Khademali, with proposal number IR.AJUMS.REC.1397.419 and IRCT code IRCT20190222042803N1. We would like to thank the Clinical Research Development Unit, Imam Khomeini Hospital, Ahvaz Jundishapur University of Medical Sciences, Ahvaz, Iran, for their cooperation.

\section{Footnotes}

Authors' Contribution: Study concept and design: Farahzad Janatmakan and Mohsen Khorami. Analysis and interpretation of data: Hojjatollah Khademali. Manuscript preparation: Fatemeh Javaherfrooshzadeh and Hojjatollah Khademali. Collection of data: Hojjatollah Khademali. Critical revision: Sara Jarirahmadi.

\section{Clinical Trial Registration Code:}

IRCT20190222042803N1

Conflict of Interests: The authors of this manuscript have no conflict of interest. 


\section{CONSORT 2010 Flow Diagram}

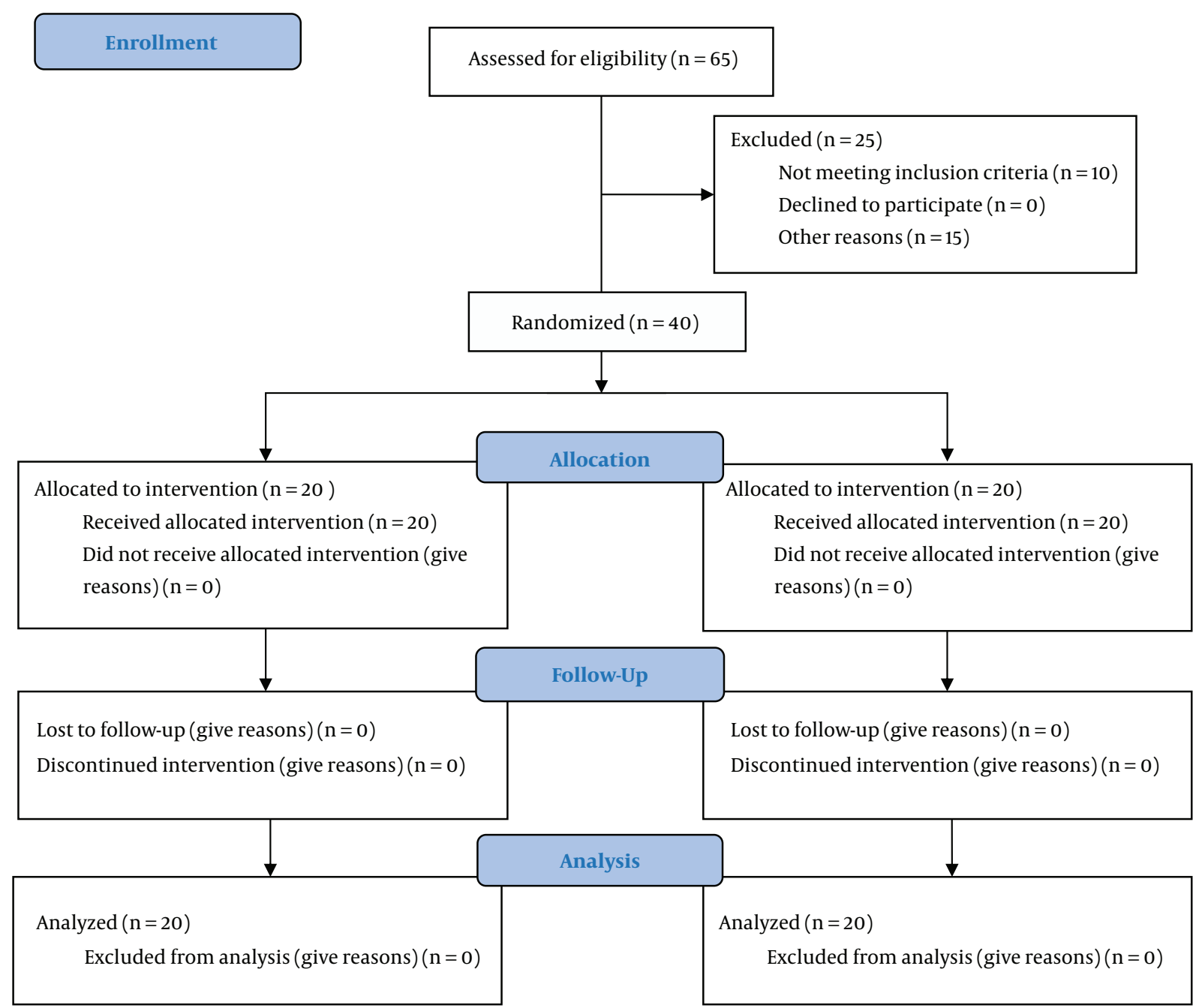

Figure 2. CONSORT 2010 Flow Diagram

Ethical Approval: This study was approved by the Ethics Committee of Ahvaz Jundishapur University of Medical Sciences (IR.AJUMS.REC.1397.419).

Funding/Support: The financial support was provided by the Vice-Chancellor for Research and Technology, Ahvaz Jundishapur University of Medical Sciences.

Informed Consent: Written informed consent was obtained by the study patients.

\section{References}

1. Safdari MR. Blood loss prevention in total knee arthroplasty (TKA): a systematic review. Rev Clin Med. 2017;4(3):102-23.
2. Hu Y, Li Q, Wei BG, Zhang XS, Torsha TT, Xiao J, et al. Blood loss of total knee arthroplasty in osteoarthritis: an analysis of influential factors.J Orthop Surg Res. 2018;13(1):325. doi:10.1186/s13018-018-1038-0. [PubMed: 30579365]. [PubMed Central: PMC6303980].

3. Sadigursky D, Araujo LM, Fernandes RJC. Efficacy of tranexamic acid in reducing blood loss in total knee arthroplasty. Acta Ortop Bras. 2018;26(1):63-6. doi: 10.1590/1413-785220182601149210. [PubMed: 29977148]. [PubMed Central: PMC6025505].

4. Sadigursky D, Andion D, Boureau P, Ferreira MC, Carneiro RJ, Colavolpe PO. Effect of tranexamic acid on bleeding control in total knee arthroplasty. Acta Ortop Bras. 2016;24(3):131-6. doi: 10.1590/1413-785220162403149200. [PubMed: 27217813]. [PubMed Central: PMC4863860].

5. Zhang H, Chen J, Chen F, Que W. The effect of tranexamic acid on blood loss and use of blood products in total knee arthroplasty: a metaanalysis. Knee Surg Sports Traumatol Arthrosc. 2012;20(9):1742-52. doi: 10.1007/s00167-011-1754-z. [PubMed: 22065294]. 
6. Karkhanehie B, Aghajanlou M, Habiballahzadeh K. Comparison of propofol and isoflurane effect on hemorrhage during spine surgery. Avicenna J Clin Med. 2015;21(4):255-62.

7. Kelley TC, Tucker KK, Adams MJ, Dalury DF. Use of tranexamic acid results in decreased blood loss and decreased transfusions in patients undergoing staged bilateral total knee arthroplasty. Transfusion. 2014;54(1):26-30. doi: 10.1111/trf.12167. [PubMed: 23521109].

8. Gardner MJ, Demetrakopoulos D, Klepchick PR, Mooar PA. The efficacy of autologous platelet gel in pain control and blood loss in total knee arthroplasty. An analysis of the haemoglobin, narcotic requirement and range of motion. Int Orthop. 2007;31(3):309-13. doi: 10.1007/s00264-006-0174-z. [PubMed: 16816947]. [PubMed Central: PMC2267605].

9. Weisel JW, Litvinov RI. Fibrin formation, structure and properties. Subcell Biochem. 2017;82:405-56. doi: 10.1007/978-3-319-49674-0_13. [PubMed: 28101869]. [PubMed Central: PMC5536120].

10. Periayah MH, Halim AS, Mat Saad AZ. Mechanism action of platelets and crucial blood coagulation pathways in hemostasis. Int J Hematol Oncol Stem Cell Res. 2017;11(4):319-27. [PubMed: 29340130]. [PubMed Central: PMC5767294].

11. Hayakawa M, Sawamura A, Gando S, Kubota N, Uegaki S, Shimojima $\mathrm{H}$, et al. Disseminated intravascular coagulation at an early phase of trauma is associated with consumption coagulopathy and excessive fibrinolysis both by plasmin and neutrophil elastase. Surgery. 2011;149(2):221-30. doi: 10.1016/j.surg.2010.06.010. [PubMed: 20655560].

12. Godje O, Gallmeier U, Schelian M, Grunewald M, Mair H. Coagulation factor XIII reduces postoperative bleeding after coronary surgery with extracorporeal circulation. Thorac Cardiovasc Surg. 2006;54(1):26-33. doi: 10.1055/s-2005-872853. [PubMed: 16485185].

13. Fenger-Eriksen C, Ingerslev J, Sorensen B. Fibrinogen concentratea potential universal hemostatic agent. Expert Opin Biol Ther. 2009;9(10):1325-33. doi: 10.1517/14712590903193051. [PubMed: 19645632].

14. Agger P, Langhoff J, Smerup MH, Hasenkam JM. Comparison between TachoComb and TachoSil for surgical hemostasis in arterial bleeding: an animal experimental study. J Trauma. 2010;68(4):838-42. doi: 10.1097/TA.0b013e3181b1388c. [PubMed: 20386279].

15. Ghavidel AA, Mirmesdagh Y, Samiei N, Dehaki MG. Haemostatic role of TachoSil surgical patch in cardiac surgery. J Cardiovasc Thorac Res. 2014;6(2):91. doi: 10.5681/jcvtr.2014.020.

16. Javaherforoosh Zadeh F, Janatmakan F, Shafaee Tonekaboni M, Soltanzadeh M. The Effect of Fibrinogen on Blood Loss After Lumbar Surgery: A Double-Blind Randomized Clinical Trial. Anesth Pain Med. 2019;9(3). e91199. doi: 10.5812/aapm.91199. [PubMed: 31497522]. [PubMed Central: PMC6712358].

17. Javaherforoosh Zadeh F, Janatmakan F, Soltanzadeh M, Zamankhani M. Investigating the effect of fibrinogen injection on bleeding in coronary artery bypass surgery: A clinical trial. Anesth Pain Med. 2019;9(4). doi: 10.5812/aapm.92165.

18. Matonick JP, Hammond J. Hemostatic efficacy of EVARREST, Fibrin Sealant Patch vs. TachoSil(R) in a heparinized swine spleen incision model. J Invest Surg. 2014;27(6):360-5. doi: 10.3109/08941939.2014.941444. [PubMed: 25361019].

19. Rahimzadeh P, Imani F, Faiz SH, Entezary SR, Nasiri AA, Ziaeefard M. In- vestigation the efficacy of intra-articular prolotherapy with erythropoietin and dextrose and intra-articular pulsed radiofrequency on pain level reduction and range of motion improvement in primary osteoarthritis of knee. J Res Med Sci. 2014;19(8):696-702. [PubMed: 25422652]. [PubMed Central: PMC4235087].

20. Wang GJ, Hungerford DS, Savory CG, Rosenberg AG, Mont MA, Burks SG, et al. Use of fibrin sealant to reduce bloody drainage and hemoglobin loss after total knee arthroplasty: a brief note on a randomized prospective trial.J Bone Joint Surg Am. 2001;83(10):1503-5. doi: 10.2106/00004623-200110000-00007. [PubMed: 11679600].

21. Heyse TJ, Haas SB, Drinkwater D, Lyman S, Kim HJ, Kahn BA, et al. Intraarticular fibrinogen does not reduce blood loss in TKA: a randomized clinical trial. Clin Orthop Relat Res. 2014;472(1):272-6. doi: 10.1007/s11999-013-3036-1. [PubMed: 23657879]. [PubMed Central: PMC3889440].

22. Bae $\mathrm{KC}$, Cho $\mathrm{CH}$, Lee KJ, Son ES, Lee SW, Lee SJ, et al. Efficacy of intraarticular injection of thrombin-based hemostatic agent in the control of bleeding after primary total knee arthroplasty. Knee Surg Relat Res. 2014;26(4):236-40. doi: 10.5792/ksrr.2014.26.4.236. [PubMed: 25505706]. [PubMed Central: PMC4258491].

23. Kadam P, Bhalerao S. Sample size calculation. Int J Ayurveda Res. 2010;1(1):55-7. doi: 10.4103/0974-7788.59946. [PubMed: 20532100]. [PubMed Central: PMC2876926].

24. Ben-David D, Srouji S, Shapira-Schweitzer K, Kossover O, Ivanir E, Kuhn G, et al. Low dose BMP-2 treatment for bone repair using a PEGylated fibrinogen hydrogel matrix. Biomaterials. 2013;34(12):2902-10. doi: 10.1016/j.biomaterials.2013.01.035. [PubMed: 23375953].

25. Nadler SB, Hidalgo JH, Bloch T. Prediction of blood volume in normal human adults. Surgery. 1962;51(2):224-32. [PubMed: 21936146].

26. Mercuriali F, Inghilleri G. Proposal of an algorithm to help the choice of the best transfusion strategy. Curr Med Res Opin. 1996;13(8):465-78. doi: 10.1185/03007999609115227. [PubMed: 9010613].

27. Watts CD, Pagnano MW. Minimising blood loss and transfusion in contemporary hip and knee arthroplasty. J Bone Joint Surg Br. 2012;94(11 Suppl A):8-10. doi: 10.1302/0301-620X.94B11.30618. [PubMed: 23118371].

28. Levy O, Martinowitz U, Oran A, Tauber C, Horoszowski H. The use of fibrin tissue adhesive to reduce blood loss and the need for blood transfusion after total knee arthroplasty. A prospective, randomized, multicenter study. J Bone Joint Surg Am. 1999;81(11):1580-8. doi: 10.2106/00004623-199911000-00010. [PubMed: 10565650].

29. Wong J, Abrishami A, El Beheiry H, Mahomed NN, Roderick Davey J, Gandhi R, et al. Topical application of tranexamic acid reduces postoperative blood loss in total knee arthroplasty: a randomized, controlled trial. J Bone Joint Surg Am. 2010;92(15):2503-13. doi: 10.2106/JBJS.I.01518. [PubMed: 21048170].

30. Tartaglia N, Di Lascia A, Lizzi V, Cianci P, Fersini A, Ambrosi $A$, et al. Haemostasis in thyroid surgery: Collagen-fibrinogenthrombin patch versus cellulose gauze-our experience. Surg Res Pract. 2016;2016:3058754. doi: 10.1155/2016/3058754. [PubMed: 27018148]. [PubMed Central: PMC4785271].

31. Payani N, Foroughi M, Bagheri A, Rajaei S, Dabbagh A. Effect of local fibrinogen administration on postoperative bleeding in coronary artery bypass graft patients. JCMA. 2015;1(1):23-7. doi: 10.22037/jcma.vili.10597. 